\title{
The Synthesis of New Solid-phase Microextraction Adsorption Material and Its Adsorption Performance
}

\author{
Fu Shuang ${ }^{*}$, Xia Chunhui, Li Hongmei and Xu Feng \\ College of Pharmacy, Qiqihar Medical University, Qiqihar 161006, China
}

\begin{abstract}
This article uses three kinds of monomers: the styrene, butyl acrylate and Vinyltriisopropoxysilane, synthesized the solid phase micro extract coating adsorption material, carries on the structure characterization and the physical performance test. Takes it as the solid phase micro extract coating, carries on the inspection to its adsorbability, the experiment obtains the detection limit of the benzene which is $0.21 \mu \mathrm{g} / \mathrm{L}$; The detection limit of the toluene is $30 \mu \mathrm{g} / \mathrm{L}$; The detection limit of the $\mathrm{m}$-Xylene is $0.55 \mu \mathrm{g} / \mathrm{L}$. Indicated the adsorptivity of this polymer has more effection toward the small molecular aromatic hydrocarbon as the solid phase micro extract coating adsorption material, and the limit of detection is lower compared to the commodity extract coating, there are some promotion and adoption value.
\end{abstract}

Keywords: Adsorptivity, solid phase micro extract coating, structure attribute.

\section{INTRODUCTION}

The solid phase micro extract (Solid-Phase microextraction, SPME) is the most popular sample pretreatment method in the chromatograph analysis, this method is a combination of extraction, concentration, desorption and sample injection, the sensitivity is high and the operation is simple, the solid phase micro extract technology is coating the organic high polymer material on a quartz fiber surface as the extract coating, then extract the organic molecules and do the preenrichment, carries on the hot desorption directly in the gas chromatography sample injector, increased the analysis speed and the sensitivity. The coating is the core of the solid phase micro extract technology, a variety of new coating material and the preparation technology which come recently years has further opened up the solid phase micro extract technology application scope, synthesized the new solid phase micro extract adsorption material is the topic which the very many scientific research worker craved [1-6]. The literature [7] synthesis polymers, served as the solid phase micro extract coating the adsorption material which combining with the quartz fiber well and cohesiveness are good, can add this polymer as the solid phase micro extract coating in the benzene with water or the toluene and the adsorption is good, but xylene adsorptivity is not very ideal, myself tried to find out the polymer monomer, the polymerization proportion and the experimental condition once more and finally obtain the phenylethylene-n-butyl acrylate-Vinyltriisopropoxysi-lane terpolymer as the solid phase micro extract adsorption material, and takes it as the solid phase micro extract coating to determine the adsorptivity to the Benzenes, the determination result demonstrated this polymer has the better adsorptivity to the

*Address correspondence to this author at the College of Pharmacy, Qiqihar Medical University, Heilongjiang, 161006, P.R. China; Tel: 15846289920; E-mail: fsjt1980@163.com benzene class aromatic hydrocarbon, it worth the further research and the promotion.

\section{EXPERIMENTAL PART}

\subsection{Main Instruments and Reagent}

GC-122 gas phase chromatograph, flame ionization detector (Shanghai Exact sciences Limited company); Nicolet 380 Fourier transformation infrared spectroscope (Shanghai kui Yuan scientific instrument limited company); Pyris D iamond TG /DTA thermal analysis meter (American PE Corporation); Scanning probe microscope (Shanghai Zhuo Lunwei nanometer science and technology limited company); Euler viscosity medicinal preparation (Shanghai Ping Xuan Scientific instrument Limited company); $20 \mathrm{~mL}$ screwtop extractionflask (Dongguan spectrum sign experiment equipment science and technology limited company); The building-up reactions installment (assembles voluntarily); Styrene (AR), acrylic acid Ding ester (AR), ethenyl three isopropoxy silicon hydride (AR); Nonyl phenol polyethyleneoxide ether (0P-10, AR), lauryl sodium sulfate (AR), persulfuric acid ammonia (AR), ferrous sulfate (AR); Benzene (AR), toluene (AR), m-xylol (AR).

\subsection{Building-up Reactions Principle}

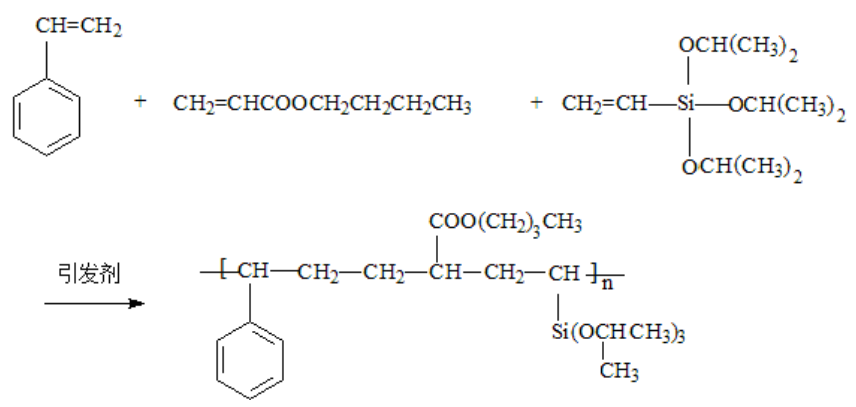

2015 Bentham Open 


\subsection{Polymer Synthesis}

Under the nitrogen protection, adds $30 \mathrm{~mL}$ aqueous solution including $1 \mathrm{~g}$ OP-10 and the $0.25 \mathrm{~g}$ lauryl sodium sulfate to the reaction bulb, adds $5 \mathrm{ml}$ styrene and $5 \mathrm{~mL}$ the butyl acrylate, emulsifies $20 \mathrm{~min}$, elevates temperature slowly to $72{ }^{\circ} \mathrm{C}$, adds $3 \mathrm{~mL} 3 \%$ persulfuric acid ammonia liquor solution, stirs $10 \mathrm{~min}$.

Add $5 \mathrm{~mL}$ styrene and $5 \mathrm{~mL}$ butyl acrylate in the separating funnel, adds by drops to the reaction bulb about $20 \mathrm{~min}$, continues to react for $1 \mathrm{~h}$. Lower the temperature of reaction bulb to $65^{\circ} \mathrm{C}$, joins $3 \mathrm{~mL} 3 \%$ ammonium persulfate and the $3 \mathrm{~mL} 0.1 \%$ ferrous sulfate water-soluble fluid, $5 \mathrm{~mL}$ styrene, $5 \mathrm{~mL}$ butyl acrylate and $1.2 \mathrm{~mL}$ Vinyltriisopropoxysilane mixed monomer, adds by drops with the separating funnel to the reaction bulb in $0.5 \mathrm{~h}$, continues to react for 1h. Rise the reaction bulb temperature to $78{ }^{\circ} \mathrm{C}$ and continue to react with the constant temperature for $1 \mathrm{~h}$. After the reaction finished, use the methyl alcohol to Precipitthe polymer, after the lavation and the vacuum drying finally obtain the white solid with viscoelasticity.

\subsection{The Structure of the Polymer Characterization}

Take $0.5 \mathrm{~g}$ polymer dissolved in $5 \mathrm{ml}$ Tetrahydrofuran solution, take a little coated in Infrared salt pieces, analysis the structure of the polymer by doing the infrared spectrogram, ascertain the exist of styrene and butyl acrylate. Analyzed the polymer with scanning electron microscopy, $\mathrm{X}$-ray probe analysis method, prove the existing way of vinyltriisopropoxy silane in the polymer.

\subsection{The Test of the Highest use Temperature of the Polymer}

Accurate take 50-60mg the polymer, put in the platinum plate, let the plate hanged inside the furnace(do not touch the furnace wall),add farmar to make the balance come to balance, set the temperature range of $20^{\circ} \mathrm{C}--600^{\circ} \mathrm{C}$; set the heating rate of $5^{\circ} \mathrm{C} / \mathrm{min}$; Use pyris Diamond TG/DTA to test it, determine the highest temperature.

\subsection{The Test of the Intrinsic Viscosity of Polymer}

When the chemical composition, solvent and temperature of polymer is determined, viscosity value associated only with the molecular weight of polymer. $[\eta]=K M \alpha([\eta]$ is intrinsic viscosity, $\mathrm{M}$ is viscosity average molecular weight, $\mathrm{K}$ and a are constant), the intrinsic viscosity of the visible polymer compounds can be reacted by it's viscosity average molecular weight .

The preparation of the solution: weight the polymer $0.25 \mathrm{~g}$ (accurate to $0.0001 \mathrm{~g}$ ), dissolved it with Tetrahydrofuran solution, constant-volume in $25 \mathrm{ml}$ volumetric flask, shake well, set aside .

The test of the out of time of the solvent: adjust the thermostatic bath to $25^{\circ} \mathrm{C}$. On viscometer tube top two sets of medical rubber tube, put in constant temperature water tank, let the capillary is perpendicular to the surface, and the water immersion on line. Move to take $10 \mathrm{ml}$ of Tetrahydrofuran solution into the viscometer, with suction ears ball to suction to ball line two centimeters of above, then loosen the suction ears to make it free fall, write down the time the solution flows between the two scribed line, that's out of time .Repeat more than three times, the error is less than 0.2 seconds, take the average value as the $t 0$.

The test of the out of time of the solution: moves $10 \mathrm{ml}$ of polymer solution injected in viscosity meter with a pipette, then prescribed it's viscosity as 1.Measured the out time as $\mathrm{t} 1$, repeat three times and take the average ;moves $5 \mathrm{ml}$ Tetrahydrofuran solution again, and the viscosity of the polymer in viscometer becomes $2 / 3$, mix it with the suction ear ball updraught, measure it and get $\mathrm{t} 2$. As the same way, add $5 \mathrm{ml}, 10 \mathrm{ml}, 15 \mathrm{ml}$ tetrahydrofuran in turn, it's viscosity is $1 / 2,1 / 3,1 / 4$, and get $\mathrm{t} 3, \mathrm{t} 4$, $\mathrm{t} 5$, and calculation $\eta \mathrm{sp} / \mathrm{C} 、 \mathrm{Ln} \eta \mathrm{r}$ by intrinsic viscosity of extrapolation method $[\eta]$.

\subsection{The Test of Capacity and Heat Resistance Performance of Polymer and Silica Fiber}

The test of the ability of combined with quartz fiber: dissolved the polymer in tetrahydrofuran, make it into the dilute solution, after several times coating and drying, make it come to 100um thickness of quartz fiber extraction. And then, put the extraction head at $70^{\circ} \mathrm{C}$ water cut the top of the empty bottle top, takes it out after $1 \mathrm{~h}$, hangs it in the middle of $300^{\circ} \mathrm{C}$ oven, $1 \mathrm{~h}$ 's late, to observe the status of the coating, then put the extraction head into water again, last $1 \mathrm{~h}$ at room temperature. And then hangs it in the middle of $300^{\circ} \mathrm{C}$ oven, $1 \mathrm{~h}$ 's late, to observe the status of the coating.

The test of thermal performance: coupled extraction head with gas chromatograph to investigate the thermal stability of the coating. Install a 2 meters long empty chromatographic on the gas chromatograph, set the initial temperature of the gasification chamber as $100^{\circ} \mathrm{C}$, set the termination temperature as $240^{\circ} \mathrm{C}$, the rate of heating is $5^{\circ} \mathrm{C}$ every 5 minutes, set the oven temperature as $200^{\circ} \mathrm{C}$, set the FID detector temperature as $240^{\circ} \mathrm{C}$, observe the change of chromatographic peak baseline, to realize the thermal stability of the coating.

\subsection{Self-made Solid-phase Microextraction Device}

Homemade SPME device: Take a $15 \mathrm{~cm}$ long optical quartz fiber, get ride of the layer on the surface of the polyester with concentrated sulfuric acid, and then inserted the quartz fiber $0.25 \mathrm{~kg} / \mathrm{L}$ polymer in tetrahydrofuran solution coating repeatedly, make it become $1 \mathrm{~cm}$ long and $100 \mathrm{um}$ thick coating, combine it with the $5 \mathrm{uL}$ microsyringe into the device of 5L SPME.

Device as shown in Fig. (1).

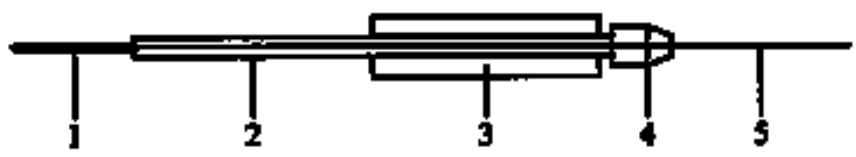

Fig. (1). Self-made solid-phase microextraction device.

(1-coating 2-outer casing 3-Glass syringe casing 4-Sealed knob 5Quartz fiber and inner casing). 


\subsection{The Test of Adsorption Ability of Polymer Coating of Benzene Class Aromatics}

\subsubsection{The Chromatographic Conditions}

The chromatographic column is $2 \mathrm{~m} \times 3 \mathrm{~mm}$ i.d. Stainless steel column; and acid red supporter filled within $8 \%$ SE$30 / 202(60-80 \mathrm{mesh})$; the temperature of injection port is $190^{\circ} \mathrm{C}$; the column temperature is $190^{\circ} \mathrm{C}$; the detector temperature is $220^{\circ} \mathrm{C}$; the sensitivity is 1010 ; the attenuation is 0 ; the nitrogen as carrier gas; the flow rate of the carrier gas is $40 \mathrm{ml} / \mathrm{min}$; the flow rate of the hydrogen is $40 \mathrm{ml} / \mathrm{min}$; the flow rate of the air is $400 \mathrm{ml} / \mathrm{min}$.

\subsubsection{The Conditions of Extraction}

The extraction of head: $100 \mu \mathrm{m}$; The extraction bottle: $15 \mathrm{ml}$; The extract content: $5 \mathrm{ml}$; The extraction temperature: $45^{\circ} \mathrm{C}$ (water bath); The equilibrium time:25min; The extraction time: 25min; The dosage of salting-out agent $(\mathrm{NaCl})$ : $0.5 \mathrm{~g}$; The desorption temperature: $190^{\circ} \mathrm{C}$; The desorption time:2min; keep the bath temperature and the stirring speed are constant.

\section{THE RESULTS AND DISCUSSION}

\subsection{The Choice of the Conditions of Polymer Synthesis}

Based on the thermal stability of polymers, and fiber bonding force, viscoelasticity as the main factors to test the target extraction ability, the optimal reaction conditions determined by the orthogonal experiment method is: the volume ratio of styrene and butyl acrylate is $1: 1$; the mass fraction of vinyl silane three isopropyl oxygen radicals to monomer is $5 \%$; the temperature of adding organic silicon is $65^{\circ} \mathrm{C}$; the mass fraction of initiator $(\mathrm{NH} 4) 2 \mathrm{~S} 2 \mathrm{O} 4$ is $3 \%$; the mass fraction of $\mathrm{FeSO} 4$ is $0.1 \%$; under this condition the production rate is $74 \%$.

\subsection{The Structure Characterization of the Polymer}

The test results carried out on the polymer by infrared spectrum scanning is as shown in Fig. (2).

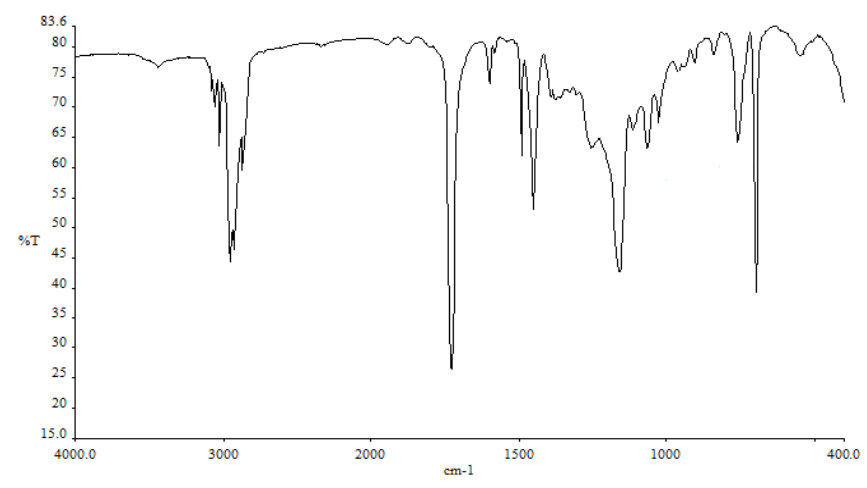

Fig. (2). The infrared spectrum scanning of the polymer.

Through analysis the infrared spectrum, in $1600 \mathrm{~cm}-1$, $1450 \mathrm{~cm}-1,1453 \mathrm{~cm}-1$ (the vibration of benzene ring skeleton) and $695 \mathrm{~cm}-1$ (aromatic hydrogen plane bending vibration) absorption proves the existence of benzene; in $1680-1620 \mathrm{~cm}-1$ has no absorption peak of $\mathrm{C}=\mathrm{C}$, proves that styrene has polymerization; in the $1731 \mathrm{~cm}-1 \quad(\mathrm{C}=\mathrm{O}$ stretching vibration) and $1162 \mathrm{~cm}-1 \quad(\mathrm{C}=\mathrm{C}-\mathrm{O}$ stretching vibration), the strong absorption peak is the characteristic of ester absorption, and proves the existence of ester, in $1066 \mathrm{~cm}-1$ (Si-O-C stretching vibration) and $963 \mathrm{~cm}-1$ (Si-C stretching vibration) has absorption peak, proved that the existence of organic silicon.

The results of analysis the polymer carried out by scanning electron microscopy, X-ray probe is as shown in Fig. (3).

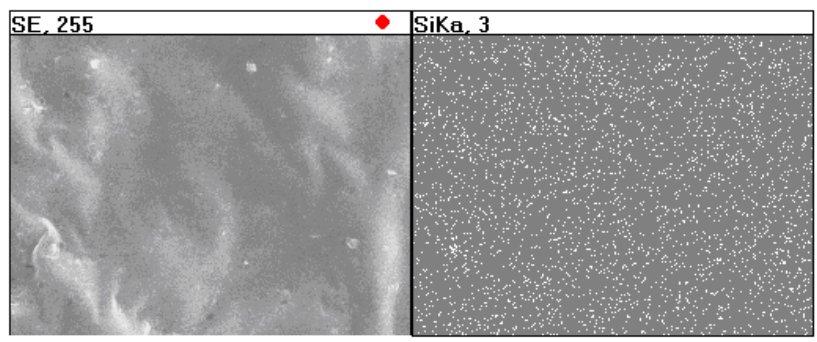

Fig. (3). The analysis diagram of scanning electron microscopy,Xray probe of polymer.

Though electron microscopy, X-ray probe we can analysis this polymer silicon is evenly distributed. Further proves that the polymer vinyl silane, three isopropyl oxygen radicals is converged on styrene and butyl acrylate polymer rather than on the adsorption, further evidence that the polymer is the result of three kinds of monomer polymerization.

An analysis to result of the thermal performance test on polymer is as shown in Fig. (4).

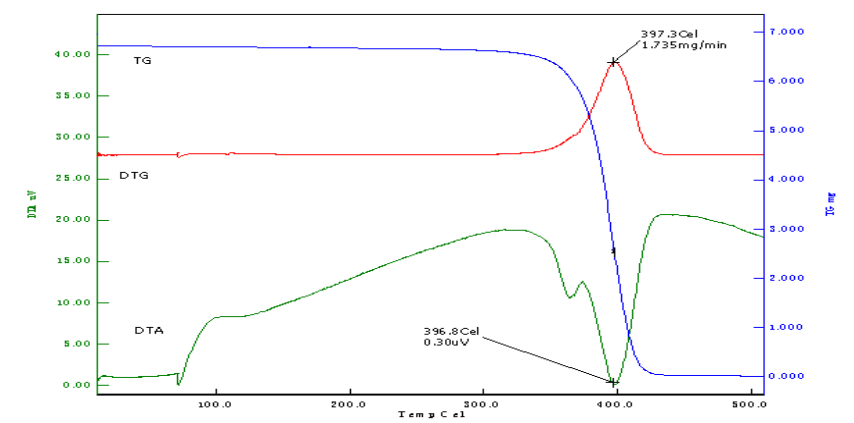

Fig. (4). The curve of polymer's thermogravimetric/differential thermal.

By thermogravimetric/differential thermal curve analysis shows that the highest temperature of the polymer can be set to $330^{\circ} \mathrm{C}$, and the transition temperature of the polymer glass transition is $65^{\circ} \mathrm{C}$.

An analysis to result of the intrinsic viscosity determination of polymer is as shown in Fig. (5).

By Iim $\mathrm{sp} / \mathrm{C}=\operatorname{IimLn\eta } \mathrm{r} / \mathrm{C}=[\eta]$, we can know that when $\mathrm{C} \rightarrow 0, \eta \mathrm{sp} / \mathrm{C}$ and $\mathrm{Ln} \eta \mathrm{r} / \mathrm{C}$ to a point which is $[\eta]$. Determine $\eta \mathrm{sp} / \mathrm{C}$ and $\mathrm{Ln} \eta \mathrm{r} / \mathrm{C}$ to the point that is $\mathrm{y}=1.6971$, namely the

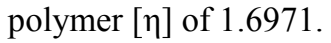

\subsection{An Analysis to Results of Polymer Coating Combined with the Ability to Quartz Fiber and Thermal Performance Test}

Combined with quartz fiber capacity test results: according to section 1.7, repeating 20 times, the polymer 
coating has no obvious change in the appearance. It is visible that the polymer and fiber binding force strong, good filmforming, high and low temperature resistance, resistance to water.

\subsection{An Analysis to Results of Polymer Coating Combined with the Ability to Quartz Fiber and Thermal Performance Test}

Combined with quartz fiber capacity test results: according to section 1.7 , repeating 20 times, the polymer coating has no obvious change in the appearance. It is visible that the polymer and fiber binding force strong, good filmforming, high and low temperature resistance, resistance to water.

The results of thermal performance test: when the gasification chamber temperature to $240^{\circ} \mathrm{C}$, the extraction coating doesn't change; and the chromatography workstation baseline is very stable, that is conform to the requirements of the solid phase microextraction and heat-resistant coating performance conditions.

3.4. An Analysis to the Result of the Adsorption Performance Test Uses for the Self-made Solid-phase Microextraction Device to Substance Such as Benzene

Take six $100 \mathrm{ml}$ volumetric flasks, compound containing benzene, toluene, xylene between $0,10,20,30,40$ and 50 $\mu \mathrm{g} / \mathrm{L}$ aqueous solution, application of self-made solid-phase microextraction device between benzene, toluene, xylene $0.00 \mu \mathrm{g} / \mathrm{L} \sim 50.00 \mu \mathrm{g} / \mathrm{L}$ range for headspace extraction, were measured three times, the curve equation of linear regression and correlation coefficient respectively: benzene $\mathrm{h}=0.0077$ $+0.0085 \mathrm{c}, \mathrm{r}=0.9926$, the lowest detection limit is $0.21 \mu \mathrm{g} / \mathrm{L}$; Toluene $\mathrm{h}=0.00570 .0057, \mathrm{r}=0.9890$, the lowest detection limit is $0.30 \mu \mathrm{g} / \mathrm{L}$; Meta-xylene $\mathrm{h}=0.0047+$ $0.0519 \mathrm{c}, \mathrm{r}=0.9793$, the lowest detection limit is $0.55 \mu \mathrm{g} / \mathrm{L}$, the measured chromatograms and linear relationship between three kinds of materials are as shown in Fig. (5), Fig. (6).

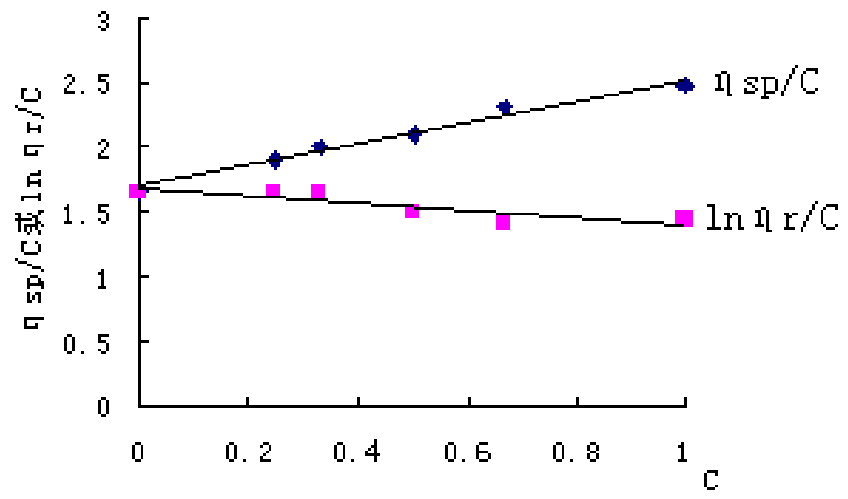

Fig. (5). The polymer $\eta \mathrm{sp} / \mathrm{C}$ of $\mathrm{C}$ and $\mathrm{Ln} \eta \mathrm{r} / \mathrm{C}$ of $\mathrm{C}$ diagram.

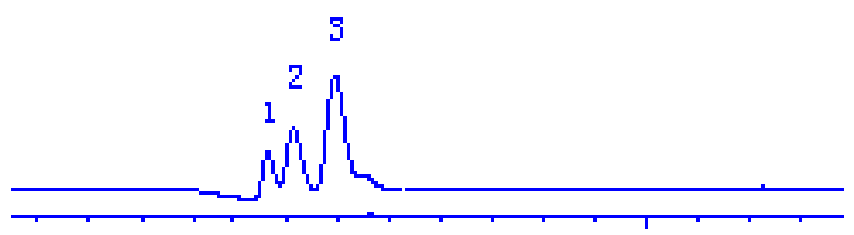

Fig. (6). The chromatograms of the self-made solid-phase microextraction device for the determination of the water containing benzene, toluene, xylene.

(the peak 1 : Benzene; the peak 2: toluene; the peak 3: metaxylene).

As a result, the coating of benzene, toluene and metaxylene SPME GC extraction effect is good, and high sensitivity; comparing with goods extraction head which is reported in the literature [7], its effect is better than that of PDMS and PA goods extraction head.

The test of the precision and the detection limit is as shown in Table $\mathbf{1}$.

\subsection{Compared with Goods Extraction Head}

Compared with goods extraction head, the self-made extraction head (100 Lm) and goods extraction head (including $100 \mu \mathrm{m}$ PDMS and $85 \mu \mathrm{m}$ PA) extract and

Table 1. Precision and detection limitof the method.

\begin{tabular}{|cccc|}
\hline Compound & Found $(\boldsymbol{\mu g} / \mathbf{L})$ & RSD $(\%, \mathbf{n = 6})$ & Dtection limit( $\boldsymbol{\mu g} / \mathbf{L})$ \\
\hline \hline Benzene & 8.75 & 0.94 & 0.21 \\
\hline Toluene & 21.17 & 1.16 & 0.30 \\
\hline xylene & 16.29 & 1.08 & 0.55 \\
\hline
\end{tabular}

Table 2. Comparison of coating.

\begin{tabular}{|cccc|}
\hline Compound & Peak area A(mm2) & Peak area A(mm2) & Peak area A(mm2) \\
\hline & Our coating & PDMS & 0.21 \\
\hline \hline Benzene & 8.75 & 0.94 & 0.30 \\
\hline Toluene & 21.17 & 1.16 & 0.55 \\
\hline xylene & 16.29 & 1.08 & 0.5 \\
\hline
\end{tabular}


chromatography analysis of the preparation of the mixed sample solution (concentration respectively $10 \mu \mathrm{g} / \mathrm{L}$ ). The chromatographic peak area (A $\mathrm{mm} 2$ ) measured were compared, the determination results are shown in Table $\mathbf{2}$.

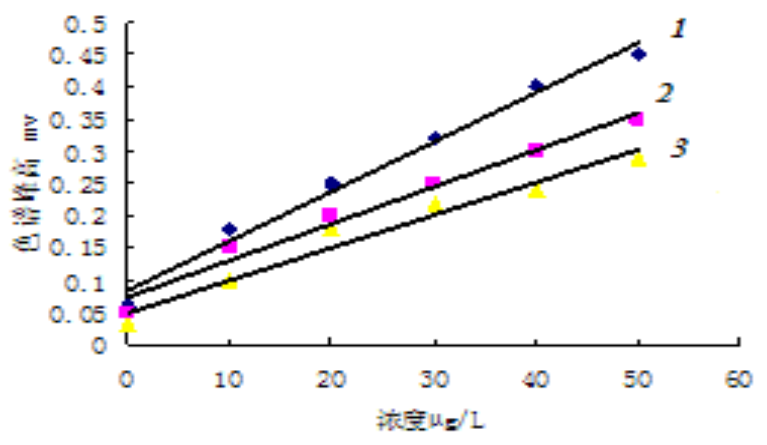

Fig. (7). Methods of linear relationship.

(the peak 1: Benzene; the peak 2: toluene; the peak 3: metaxylene)

\section{CONCLUSION}

With the monomer of styrene, butyl acrylate and Vinyl silane three isopropyl oxygen radicals, synthetic ternary copolymer. Combining the polymer with fibers to make solid phase microextraction coating, the experiments show that this coating has good thermal stability, and fiber binding force strong, good film forming, good flexibility, high and low temperature resistance, water resistance, strong for aromatics adsorption force. Application of more than one hundred times, it does not have falls off phenomenon, and it has certain promotion and use value.

\section{CONFLICT OF INTEREST}

The authors confirm that this article content has no conflict of interest.

\section{ACKNOWLEDGEMENTS}

In this paper, the research was sponsored by the Science and Technology Research Project of Heilongjiang Province Education Department (Project No. 12541932).

\section{REFERENCES}

[1] L. Yan, Q. Zhang, W. Zhang, Y. Feng, L. Zhang, T. Li, and Y. Zhang, "Hybrid organic-inorganic phenyl monolithic column for capillary electrochromatography," Electrophoresis, vol. 26, no. 15, pp. 2935-2941, 2005.

[2] D. Panavaite, A. Padarauskas, and V. Vickackaite, "Silicone glue coated stainless steel wire for solid phase microextraction," Analytica Chimica Acta, vol. 57, no. 1, pp. 45-50, 2006.

[3] W. Guan, F. Xu, W. Liu, J. Zhao, and Y. Guan, "A new poly(phthalazine ether sulfone ketone)-coated fiber for solid-phase microextraction to determine nitroaromatic explosives in aqueous samples," Journal of Chromatography A, vol. 1147, pp. 59-65, 2007.

[4] J. Chen, J. Zeng, and W. Chen, "Development of new coating for solid phase microextraction," Progress in Chemistry, vol. 21, no. 9, pp. 1922-1928, 2009.

[5] J. López-Darias, V. Pino, Y. Meng, J.L. Anderson, and A.M Afonso, "Utilization of a benzyl functionalized polymeric ionic liquid for the sensitive determination of polycyclic aromatic hydrocarbons; parabens and alkylphenols in waters using solidphase microextraction coupled to gas chromatography-flame ionization detection," Journal of Chromatography A, vol. 1217, no. 46, pp. 7189-7197, 2010.

[6] X. Liang, F. Zhao, Y. Ma and B. Zeng, "Electrochemical preparation of polypyrrole-ionic liquid coating for solid phase microextraction and its application in gas chromatographic determination of benzene derivatives," Chinese Journal of Analytical Chemistry, vol. 40, no. 10, pp. 1579-1583, 2012.

[7] S. Shen, W. Zhang, and S. Fu, "Preparation phenylethylenebutylacrylate $\mathrm{v}$ inyltriethoxy siloxane terpolymer for solid phasem icroextraction coating," Chinese Journal of Analytical Chemistry, vol. 35, no. 6, pp. 865-868, 2007.

(C) Shuang et al.; Licensee Bentham Open.

This is an open access article licensed under the terms of the Creative Commons Attribution Non-Commercial License (http://creativecommons.org/licenses/by-nc/3.0/) which permits unrestricted, non-commercial use, distribution and reproduction in any medium, provided the work is properly cited. 